http://dx.doi.org/10.35381/r.k.v5i3.913

\title{
Evaluación de riesgo y gestión administrativa en organizaciones públicas
}

\section{Risk assessment and administrative management in public organizations}

\author{
Sebastián Mauricio Ormaza-Rodríguez \\ sebastian.ormaza.17@psg.ucacue.edu.ec \\ Universidad Católica de Cuenca, Cuenca \\ Ecuador \\ https://orcid.org/0000-0002-9998-3670 \\ Johanna Rosalí Reyes-Reinoso \\ ireyesr@ucacue.edu.ec \\ Universidad Católica de Cuenca, Cuenca \\ Ecuador \\ https://orcid.org/0000-0001-82384367 \\ Fidel Edmundo Cepeda-Luna \\ fcepedal@ucacue.edu.ec \\ Universidad Católica de Cuenca, Cuenca \\ Ecuador \\ https://orcid.org/0000-0003-3858-7030 \\ Mireya Magdalena Torres-Palacios \\ mireya.torres@ucacue.edu.ec \\ Universidad Católica de Cuenca, Cuenca \\ Ecuador \\ https://orcid.org/0000-0002-7724-3313
}

Recibido: 20 de mayo de 2020 Revisado: 15 de junio de 2020 Aprobado: 30 de julio de 2020 Publicado: 15 de agosto de 2020 


\title{
RESUMEN
}

El objetivo fue generar una evaluación de riesgo y gestión administrativa de la Empresa Municipal de Agua Potable, Alcantarillado y Saneamiento de Azogues - Ecuador (EMAPAL EP). Metodológicamente de tipo descriptiva con diseño de campo no experimental, siendo la muestra poblacional 116 funcionarios. Se observa las debilidades de la empresa en cuanto a la movilización restringida por falta de vehículos de propiedad de la institución con un $37.50 \%$ de inconformidad y a la vez la segunda debilidad que se puede identificar es la falta de coordinación por parte de departamentos con un $36.90 \%$ de insatisfacción por parte de los funcionarios. Se establece una realidad y la importancia para la utilización de la evaluación de riesgos y gestión administrativa, por cuanto es una herramienta utilizada por las empresas y ayuda a detectar las debilidades, con ello se puede mejorar la calidad, un mejor desempeño para satisfacción de clientes internos y externos.

Descriptores: Administración de empresas; productividad; calidad del agua; calidad de la vida laboral. (Palabras tomadas del Tesauro UNESCO).

\begin{abstract}
The objective was to generate a risk assessment and administrative management of the Municipal Company of Potable Water, Sewerage and Sanitation of Azogues - Ecuador (EMAPAL EP). Methodologically descriptive with a non-experimental field design, the population sample being 116 officials. The weaknesses of the company in terms of restricted mobilization due to the lack of vehicles owned by the institution are observed with a $37.50 \%$ disagreement and at the same time the second weakness that can be identified is the lack of coordination by departments with a $36.90 \% \%$ dissatisfaction on the part of officials. A reality and importance is established for the use of risk assessment and administrative management, since it is a tool used by companies and helps to detect weaknesses, with this quality can be improved, better performance for customer satisfaction internal and external.
\end{abstract}

Descriptors: Business management; productivity; water quality; quality of working life. (Words taken from the UNESCO Thesaurus). 


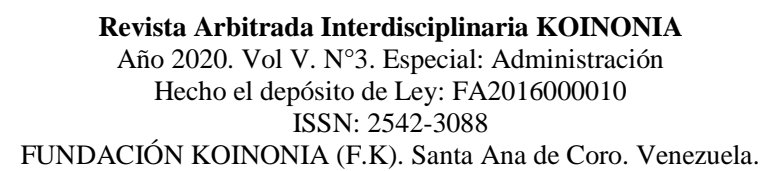

Sebastián Mauricio Ormaza-Rodríguez; Johanna Rosalí Reyes-Reinoso; Fidel Edmundo Cepeda-Luna; Mireya Magdalena Torres-Palacios

\section{INTRODUCCIÓN}

La planeación estratégica dentro de una empresa constituye el proceso administrativo, un sistema global gerencial en el que realce el "que lograr" el "qué hacer". En la planificación Estratégica busca determinar los objetivos posibles de alcanzar y en qué negocio o área concursar, aprovechando las amenazas y oportunidades que el entorno ofrece, de manera para que sea estrictamente practica y a la misma vez eficaz, tomarse en cuenta que se tiene que adaptar a las reacciones de las personas a quienes puedan afectar (Castaño-Ramírez, 2011).

En este sentido, la Empresa Pública del Agua EPA EP, posee el plan estratégico empresarial 2017-2021), derivandose el en la Empresa Municipal de Agua Potable, Alcantarillado y Saneamiento de Azogues (EMAPAL EP), el plan estratégico 2020, el cual posee tres tipos de fases: La elaboración de un pensamiento estratégico para la empresa, donde estén identificados la Misión, Visión, valores y la estrategia donde se observe las actitudes y trabajos en nuestras actitudes en las relaciones personales, con los clientes y dentro de la empresa para enfrentar problemas a futuro que puedan darse.

Como segunda fase tenemos la definición de un plan estratégico para los siguientes 12 años donde podamos observar los temas de más alto riesgo que tenemos que superar y todos los objetivos que debemos cumplir. La tercera fase tenemos un plan de metas a mediano y corto plazo que debemos cumplir de forma anual y la responsabilidad que debe cumplir la empresa EMAPAL tanto la gerencia y directores departamentales.

En el plan estratégico de la Empresa Pública Municipal de Agua Potable y Alcantarillado de Azogues, aspira cumplir precisamente con las expectativas quienes conformaron parte de este proceso. Se piensa que el documento sea una guía eficaz que contemple las líneas de acción eficientes para que así en un futuro sea prioritario para nuestra empresa de agua. Como es de conocimiento este plan es abierto para poder realizar los cambios pertinentes que se requiera si es que es necesario si las circunstancias en las que se desempeña la empresa. 


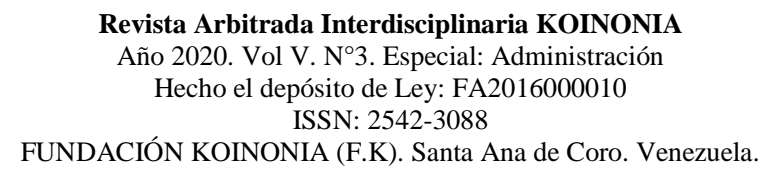

Sebastián Mauricio Ormaza-Rodríguez; Johanna Rosalí Reyes-Reinoso; Fidel Edmundo Cepeda-Luna; Mireya Magdalena Torres-Palacios

La empresa EMAPAL naciente contaba como parque automotor una retroexcavadora y una camioneta y un pequeño personal táctico y así de la misma manera un pequeño personal técnico de campo; estando al mando como su primer gerente el ingeniero Jaime Yumbla; en la época de Yumbla la primera planta de tratamiento que se operaba era la de Zhindilig con la capacidad máxima de 40lts/seg. En el mes de octubre de 1986 recién se introducen las reformas a la ordenanza de la creación de la empresa, después en los años de 1999 y 2001 se realiza la modificación para la creación de la estructura que actual, atribuciones y funciones. La empresa en los últimos 30 años no solo ha crecido si no también se ha ido fortaleciendo, ha sido gestor de obras muy importantes para la ciudadanía y sus parroquias, que con esfuerzo y gestión de su personal tanto técnicos como directivos.

Durante todos estos años el gran desafío de la empresa ha sido dotar de manera permanente el líquido vital a una población que va creciendo de manera frecuente y a alta velocidad, con sectores más altos que las plantas de tratamiento, aquí podemos observar un gran problema principal de las lejanas y limitadas fuentes de captaciones, el alto costo de la ampliación y mantenimiento de los sistemas de captación, tratamiento y conducción para la distribución del líquido vital, aquí se generaba reclamos de la ciudadanía y con ellos se sumaba una desondra sobre la calidad del agua que la empresa brindaba, generando la desconfianza que el agua no era utilizable para el consumo humano. (Empresa Pública del Agua EPA EP, 2016).

Las administraciones municipales y los directivos de la empresa se han esforzado de dura manera para poder superar estas limitaciones antes mencionada y para dar mayor confianza a la ciudadanía respecto a la calidad del servicio ofrecido, pero hasta la actualidad se mantienen dudas sobre la calidad de agua que deben ser aclaradas en su totalidad a la ciudadanía. (Empresa Pública del Agua EPA EP, 2016).

La empresa en la actualidad la empresa funciona con un amplio y moderno edificio, que con una flota vehicular bien extensa que incluye nueve camionetas, un jeep, dos retroexcavadoras, un tanquero, un hidrocleaner, dos camiones, una mini cargadora y dos 


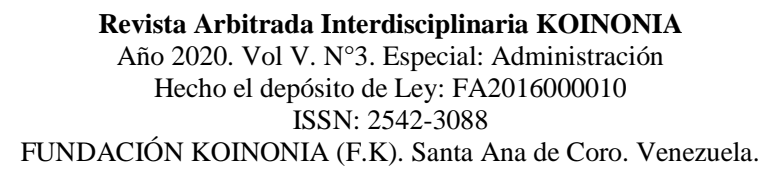

Sebastián Mauricio Ormaza-Rodríguez; Johanna Rosalí Reyes-Reinoso; Fidel Edmundo Cepeda-Luna; Mireya Magdalena Torres-Palacios

motos y quipos de alta tecnología, En el tema operativo en la actualidad se cuenta con tres plantas de tratamiento de agua potable para abarcar a toda la ciudad de líquido vital: Zhindilig, Uchupucun y Mahuarcay, siendo así sin duda por el alcance, costo y dimensión la obra de mayor importancia realizada en la vida de la empresa hasta la actualidad.

La planta de tratamiento de Uchupucun cuenta con una capacidad máxima de $901 t s / \mathrm{seg}$, es de tipo tradicional que se debe utilizar cloro gas para la desinsectación del agua. Esta planta tiene como finalidad tratar las aguas crudas de distintas captaciones; Condoryacu, el Rosario y Rubies. Entre la planta de tratamiento de Uchupucun y Mahuarcay dan servicio a la Zona alta, media, central y baja (la playa) de la ciudad de Azogues, también dicha planta sirve al sector de Uchupucun, Javier Loyola y Borrero. La planta de Zhindilig sirve a los sectores de la parte de Zhindilig, una parte de Guapan, Macas la playa y Chacapamba, su capacidad máxima de agua es de 40lts/seg.EMAPAL cuenta actualmente con seis captaciones en total.

Las captaciones de Llaucay y Nudpud, que corresponden al sector alto de la Microcuenca del Tabacay que suministra de agua a la Planta de tratamiento de Mahuarcay. La aducción se realiza mediante 8 kilómetros de canales de hormigón y con tubería. Condoryacu, Corazón Urcu, Rosario y Rubies que ubicadas en el sector bajo de la Microcuenca del Tabacay abastecen de agua a la planta de Uchupucún. Las aducciones se realizan mediante tubería. Este sistema es nuevo del año 2004. El agua tratada de las dos plantas se une y llegan al repartidor de caudales. Este consta de unos canales y compuertas donde se regulan los caudales de salida hacia las reservas. Abastece a las 5 zonas de acuerdo a lo zonificación. Estas son: Reserva de la Zona Principal, Reserva de la Zona La Playa, Reserva de la Zona Media, Reserva de la Zona de Zhapacal y Reserva de la Zona Alta. Las Redes de la Zona Principal, Alta y Media fueron construidas en el año 1987. Parte de las redes de la Zona La Playa y Zhapacal fueron construidas en el año 2004 y 2006 respectivamente. Las redes viejas de estas zonas fueron igualmente construidas en el año 1987 (Empresa Pública del Agua EPA EP, 2016). 
En función de lo planteado, el objetivo fue generar una evaluación de riesgo y gestión administrativa de la Empresa Municipal de Agua Potable, Alcantarillado y Saneamiento de Azogues - Ecuador (EMAPAL EP).

\section{Referencial teórico}

\section{Evaluación de riesgo y gestión administrativa}

La gestión administrativa es una aglomeración de ciertas actividades que se utiliza para orientar una organización mediante un comportamiento racionable de tareas, recursos y esfuerzos, cuando se tiene facultad de dominar y coordinar los distintos roles y distintas acciones que se realiza dentro de la empresa, permite identificar problemas y puede alcanzar sus objetivos, para una buena gestión administrativa en la conducción sistemática tiene como resultado una correcta obtención de datos favorables para la empresa de agua (Murillo-Vilela, et al., 2019).

El alcance de la gestión administrativa consiste en disponer de la organización y prepararla para la rápida actuación, de forma anticipada, observando todos los procedimientos y medios para poder hacer cumplir todos los objetivos y poder mermar todos los posibles problemas y efectos negativos de la empresa, para lo cual se debe basar en un proceso en la gestión administrativa que posee 4 funciones principales que son: Organización, planificación, control, dirección, (Orellana-Palomeque, et al., 2019).

\section{La gestión administrativa y sus características}

El gerente es el que está a cargo de supervisar la organización de las operaciones y garantizar que la información sea de manera verídica y que se empleen los recursos de manera eficaz, implementar el valor agregado a la empresa u organización para poder diferenciar y desarrolla los procesos para la mejora (Sanz \& López, 2018). El gerente de la empresa administrativa puede contar con el equipo de trabajo, que es conformado por los jefes departamentales, los analistas de cada departamento. El equipo de trabajo antes 
mencionado tiene ciertas responsabilidades que deben cumplir, pero con la dependencia y la especialidad que estén encargados para desempeñar, se ocupan de:

1. Liderar algunos equipos de trabajo

2. Realizar capacitaciones

3. Revisar la disponibilidad presupuestaria de la empresa u organización

4. Realizar programas para evaluar el clima laboral de la empresa

5. Controlar las remuneraciones de los trabajadores de la empresa

6. Mantener actualizado al personal

7. Elaborar normas, procedimientos y políticas.

Para la implementación de la gestión integral de los riesgos es necesario un proceso estructurado, continuo y consistente para poder tener la identificación, evaluación, reportar y medir las oportunidades y negocios, también puede medirse los con la identificación con la evaluación y medición que puede afectar a la entidad financiera de la empresa, también se implementó la estrategia de negocio (Diaz \& León, 2019).

1. Realizar un instrumento de la cobertura de los riesgos de la empresa, con una visión comprensiva del negocio.

2. Evaluar y definir las medidas de práctica proporcionadas por los riesgos.

\section{Los Riesgos inherentes a la gestión.}

Es el peligro de la probabilidad (causal de grandes pérdidas), que aparece en una determinada actividad en el transcurso de un tiempo fijado. También lo entendemos de la forma más drástica de las pérdidas que se encuentra adjunto q una operación de producción, a lo que se realiza los cambios a una planeación que se defino como estándares para poder garantizar un proceso de funcionamiento del conjunto del sistema productivo (Argudo-Tello, et al., 2019).

Por lo anterior mencionado es importante para las organizaciones analizar y verificar los riesgos más relevantes o importantes a las cueles podamos ver que se ha enfrentado y 


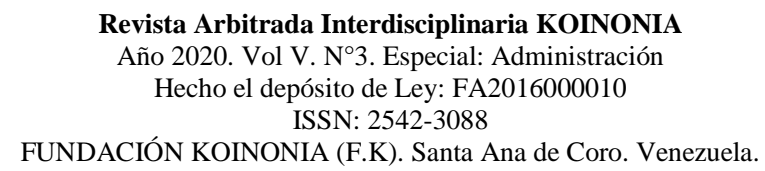

Sebastián Mauricio Ormaza-Rodríguez; Johanna Rosalí Reyes-Reinoso; Fidel Edmundo Cepeda-Luna; Mireya Magdalena Torres-Palacios

con esto pueda llevar un peligro para alcanzar sus objetivos, de mucha manera cuando la rentabilidad está ligada a los riesgos de su negocio. Cuando se identifica estos riesgos conlleva a un proceso repetitivo y de manera general se integra a la planificación estratégica y su análisis está relacionada con la actividad o el proceso y con la importancia del objetivo del riesgo, después que los riesgos son identificados de manera exacta a el nivel del organismo, se deberá ser practicado a nivel de las actividades o de los programas (Varela-Valenzuela, 2013).

Se puede mencionar que en el caso de ser una entidad, empresa u organismo financiero se requiera de manera obligatoria identificar, cuantificar y valorar la demostración de riesgo, al mismo tiempo se pretende optimizar la rentabilidad que se da de manera directa a los clientes a través de unos precios muchos más bajos y competitivos para tener mayor beneficio. (Torres-Navarro et al., 2015)

El riesgo empresarial podemos definir como un proceso que está estructurado, que es de manera consistente y de forma continua que a través del uso de algunas herramientas de reporte, medición, evaluación e identificación de los eventos que afectan principalmente el poder para alcanzar la finalidad de sus objetivos como organización. (Morón-Vásquez, et al., 2015).

La implementación de la gestión de riesgo empresarial en las organizaciones tradicionalmente se visto con fines de lucro, muy pocas veces se ha utilizado para el mejoramiento de control y para la evaluación interna de una entidad no gubernamental. Las entidades no gubernamentales se ven expuestas a bastante riesgo al igual que cualquier otra sociedad con derecho (Morón-Vásquez, et al., 2015).

Toda institución tiene un director o gerente donde cae toda su responsabilidad de la misma, por eso es de manera necesaria quien este encargado de esta función debe formarse en un líder, o por lo menos deberá tener algunas ciertas cualidades de un líder que con eso le permita ejercer su trabajo y así tener una buena relación con los trabajadores de la organización (Trógolo, et al., 2013). 


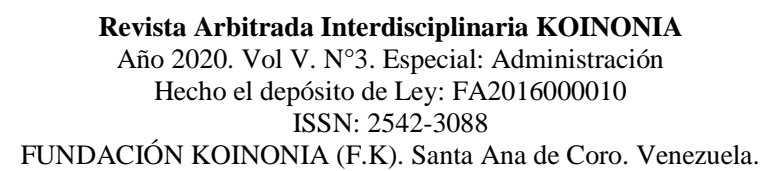

Sebastián Mauricio Ormaza-Rodríguez; Johanna Rosalí Reyes-Reinoso; Fidel Edmundo Cepeda-Luna; Mireya Magdalena Torres-Palacios

Para la gestión administrativa tiene que tener ciertos factores de importancia y exigencias legales inevitables que el titular debe tener conocimiento, o por lo menos deberá tener una buena asesoría en la parte jurídica, ya que la tarea del administrador o gerente tiene que siempre estar orientada en lo que se basa al derecho público, fabricándose así por la evolución que va tomando siempre el estado en la modernidad (Trógolo, et al., 2013). Los servicios para la realización de una administración eficaz y rigurosa de manera prolongada en el tiempo, puede producir a nivel empresarial una serie de beneficios para que se note la importancia de la empresa en su futuro tomando en cuenta algunos factores.

1. Recursos optimizados.

2. Riesgos minimizados.

3. Eficacia cuando trabajamos datos.

4. Tener un grado se seguridad para tomar las decisiones.

Hoy en día podemos observar como muchos negocios han cerrado sus puertas, tomando en cuenta que el problema principal es no saber gestionar los recursos de la empresa de forma eficaz, como principal premisa es el proceso de planificación, dirigir, organizar y controlar las labores del persona de la organización como objetivo es alcanzar las metas que se proponen para una eficaz organización de la empresa (Botero-Pinzón, 2017).

Se debe aplicar la normativa sindical, fiscal y laboral en los distintos temas de la administración, control, gestión de los recursos humanos de la empresa. También se debe aplicar la normativa que se encuentra vigente en todos los procesos de los recursos humanos. Aplicar los distintos procedimientos establecidos internos de la empresa u organización para el control y mantenimiento del fichero de la asistencia del personal. En el siglo XX en América Latina y el Caribe han tenido muchos problemas de descentralización en diversas competencias municipales. En la mayoría de países y esto hasta el día de hoy existe (Calle, et al., 2019).

Los municipios en muchos países se han consolidado a estas reformas de manera tradicional a la participación municipal en la dotación de servicios públicos que son 


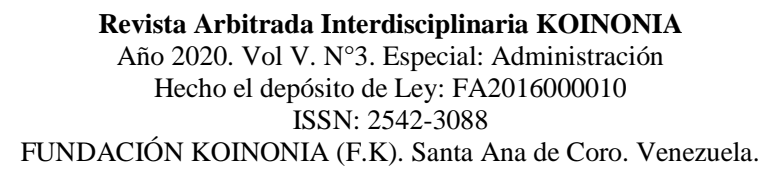

Sebastián Mauricio Ormaza-Rodríguez; Johanna Rosalí Reyes-Reinoso; Fidel Edmundo Cepeda-Luna; Mireya Magdalena Torres-Palacios

vinculadas con el agua, lo que con estos le convierten en importantes usuarios institucionales de os recursos para la ciudadanía (Casas-Cervantes, 2015). El área responsable para llevar acabo de clasificar es la gestión administrativa y de regularizar los recursos administrativos de la empresa, los recursos y procesos que tiene cada uno de los departamentos y poder ser tramitar de una mejor manera, en resumen, la gestión administrativa es la que tiene que mantener y dirigir de la mejor manera el control de los recursos de una empresa u organización para que con ello puedan obtener unos mejores resultados (Reyes, et al., 2020).

Para ejecutar de forma más factible y sencilla tenemos que modificar los procesos que faciliten la productividad en vez de atrasarlas con todos estos ajustes y modificación la productividad de la empresa se ve de una manera clara y con esto el gerente administrativo ya puede centrarse en otras distintas tareas que está a su responsabilidad (Galindo-Arevalo, 2019). Sin la implementación de una gestión administrativa no es muy probable que visualizar los objetivos, con la planificación correctamente ejecutada, un equipo que produce y organizada los objetivos que podíamos observar era inalcanzables ahora puede ser posibles a corto, mediano y largo plazo (Erazo-Álvarez \& Narváez-Zurita, 2020).

Cuando se diseñan o crean estrategias definidas todo esto puede llevar a ahorrar más dinero y poder resolver o encontrar de cierta manera un poco más inteligente a ahorrar sus servicios y productos. A lo que la estructura es mucho más organizada, los problemas se pueden arreglar de una forma correcta para satisfacer a los empleados y clientes (González-Bustos, et al., 2020). Cuando el equipo de la empresa u organización es motivado sus clientes son mucho más satisfecho. En un principio el gerente general o gerente administrativo fija metas para su empresa y con la correcta aplicación de la planificación se procese a llegar a donde se propuso para llevar todo con tranquilidad y para que esto suceda debe seguir las siguientes responsabilidades;

1. Ordenar los recursos financieros

2. Enfrentar los diferentes problemas de la empresa 
3. Analizar y resolver los problemas de la empresa

4. Verificar los diferentes aspectos para así poder prevenir los problemas

5. Se debe motivar a los empleados para que cumplan todos los procesos administrativos.

La mejor forma para realizar una muy buena gestión administrativa es visualizar los procesos que se están llevando a cabo dentro de la empresa, cuales departamentos los está llevando y quienes son las personas quien está hecho a cargo, motivarlas y capacitarles para que vayan mejorando constantemente.

\section{Procedimiento y organización}

Es indispensable saber cuáles son las necesidades de cada dirección, unidad o puestos de trabajo con el fin de mejorar las condiciones de trabajo y los recursos para que estén a gusto y satisfecho y puedan desempeñar de manera correcta sus labores. El proceso podemos definirlo como un conjunto de prácticas ya establecidas que identifica resuelve y analiza los problemas que constantemente surgen de la empresa. (Medina-León, et al., 2019).

\section{MÉTODO}

Se generó un proceso investigativo desde la tipología descriptiva de campo no experimental, siendo la muestra poblacional 116 funcionarios de la Empresa Municipal de Agua Potable, Alcantarillado y Saneamiento de Azogues (EMAPAL EP), siendo encuestados vía online mediante un cuestionario en escala Likert, siendo validado por juicio de expertos y cálculo de coeficiente Alfa de Cronbach de 0,92 siendo confiable para su aplicación, analizándose los datos obtenidos mediante estadística descriptica. 


\section{RESULTADOS}

Se pudo identificar que existe un $38 \%$ de funcionarios que identifica un problema de tecnología que afecta a la empresa en varios departamentos, ya sea por retrasos en los sistemas contables, tanto humano, facturación. También se identificó que en la dirección financiera requiere urgentemente un mejoramiento, por cuanto hay un $29.70 \%$ de inconformidad con relación a los demás departamentos, de igual manera hacen referencia a la dirección de planificación con un $28.40 \%$ de inconformidad para requerir un mejoramiento de dicho departamento, por último se observa las debilidades de la empresa en cuanto a la movilización restringida por falta de vehículos de propiedad de la institución con un $37.50 \%$ de inconformidad y a la vez la segunda debilidad que se puede identificar es la falta de coordinación por parte de departamentos con un $36.90 \%$ de insatisfacción por parte de los funcionarios.

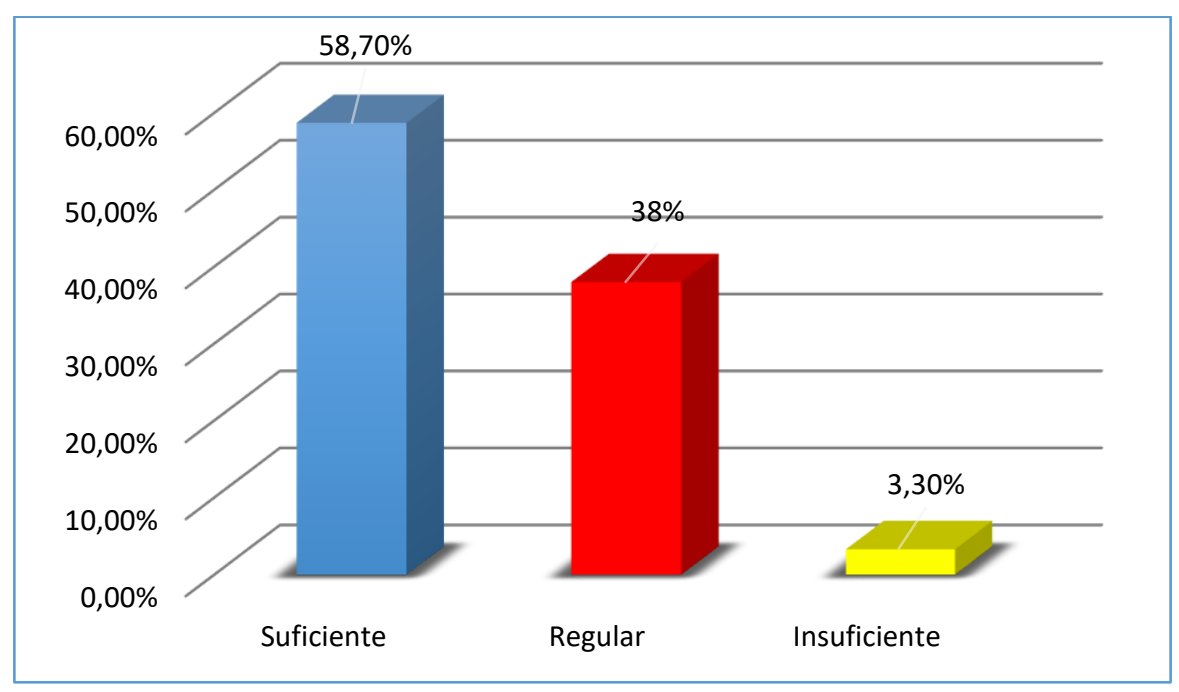

Figura 1. Evaluación del riesgo y gestión administrativa. 


\section{PROPUESTA}

A partir de los resultados que se obtuvieron se ha determinado dentro de la evaluación de riesgos y gestión administrativa, no son aplicadas de manera eficaz por parte de los distintos departamentos de la empresa ya que existen algunas inconsistencias precisamente por la falta de estructura y definición de procesos en la cual se determinen las actividades, las responsabilidades y las acciones que deben ser asumidas por cada uno de los funcionarios que pertenezcan a cada área, con base en lo expuesto se diseña un Instructivo de la Evaluación de Riesgos y Gestión Administrativa con la finalidad de agilitar la gestión interna administrativa de la empresa.

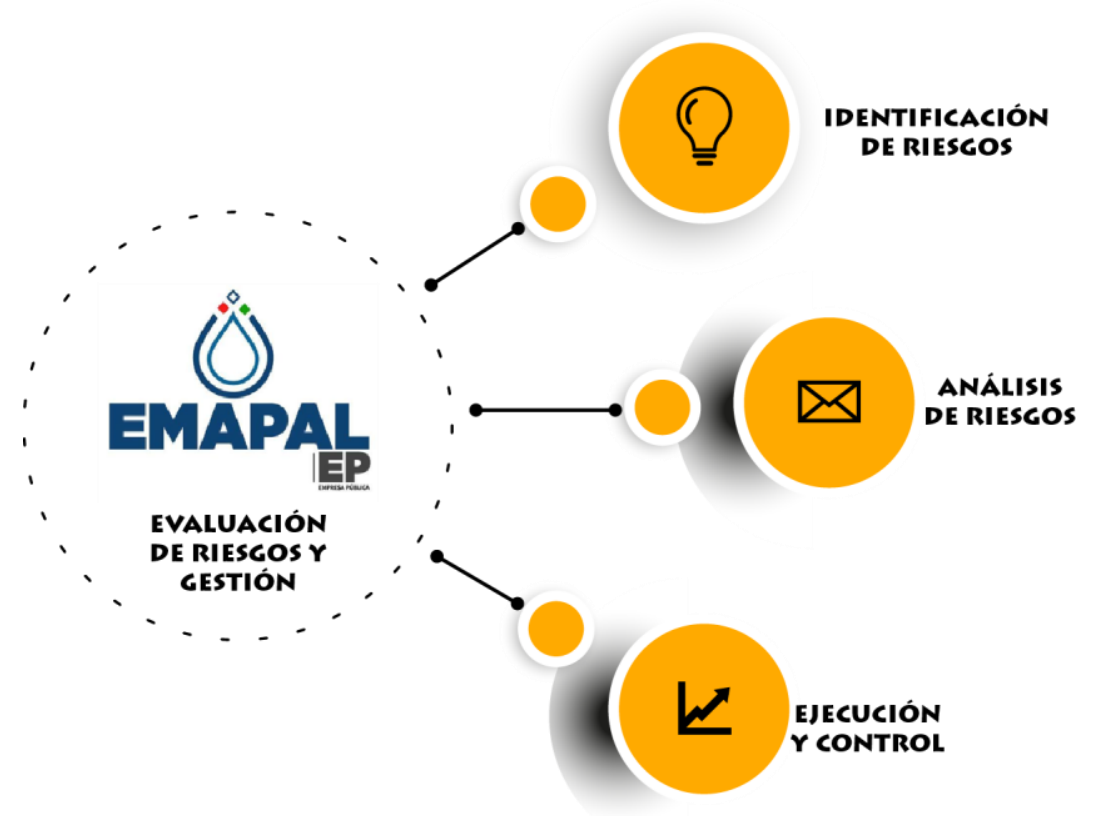

Figura 2. Esquema de la Propuesta. 


\section{Identificación del riesgo}

Se inicia con el análisis de la situación que está atravesando la empresa para identificar el riego en la actualidad a través del análisis DAFO con factores críticos para el éxito.

\begin{tabular}{|c|c|}
\hline Factores Críticos para el Éxito & PESO \\
\hline \multicolumn{2}{|l|}{ OPORTUNIDADES } \\
\hline 1. Pagos on line & $7 \%$ \\
\hline 2. Planes de Inversión & $6 \%$ \\
\hline 3. Disminución tasas de interes & $10 \%$ \\
\hline Subtotal & $23 \%$ \\
\hline \multicolumn{2}{|l|}{ AMENAZAS } \\
\hline 1. Alta rotación del TH & $8 \%$ \\
\hline 2. Crecimiento urbano & $10 \%$ \\
\hline 3. Cambio climático & $8 \%$ \\
\hline Subtotal & $25 \%$ \\
\hline \multicolumn{2}{|l|}{ FORT ALEZAS } \\
\hline 1. Talento humano calificado & $9 \%$ \\
\hline 2. Responsabilidad social & $8 \%$ \\
\hline 3. Infraestructura adecuada & $7 \%$ \\
\hline Subtotal & $24 \%$ \\
\hline \multicolumn{2}{|l|}{ DEBILIDADES } \\
\hline 1. Deficiente coordinación entre departamentos & $6 \%$ \\
\hline 2. Insuficiente nivel tecnológico & $15 \%$ \\
\hline 3. Movilidad inadecuada & $7 \%$ \\
\hline Subtotal & $28 \%$ \\
\hline TOTAL & $100 \%$ \\
\hline
\end{tabular}

Figura 3. Análisis DOFA con factores críticos para el éxito. 
Revista Arbitrada Interdisciplinaria KOINONIA

Año 2020. Vol V. N³. Especial: Administración

Hecho el depósito de Ley: FA2016000010

ISSN: 2542-3088

FUNDACIÓN KOINONIA (F.K). Santa Ana de Coro. Venezuela.

Sebastián Mauricio Ormaza-Rodríguez; Johanna Rosalí Reyes-Reinoso; Fidel Edmundo Cepeda-Luna; Mireya Magdalena Torres-Palacios

Brechas respecto al nivel esperado del diagnóstico

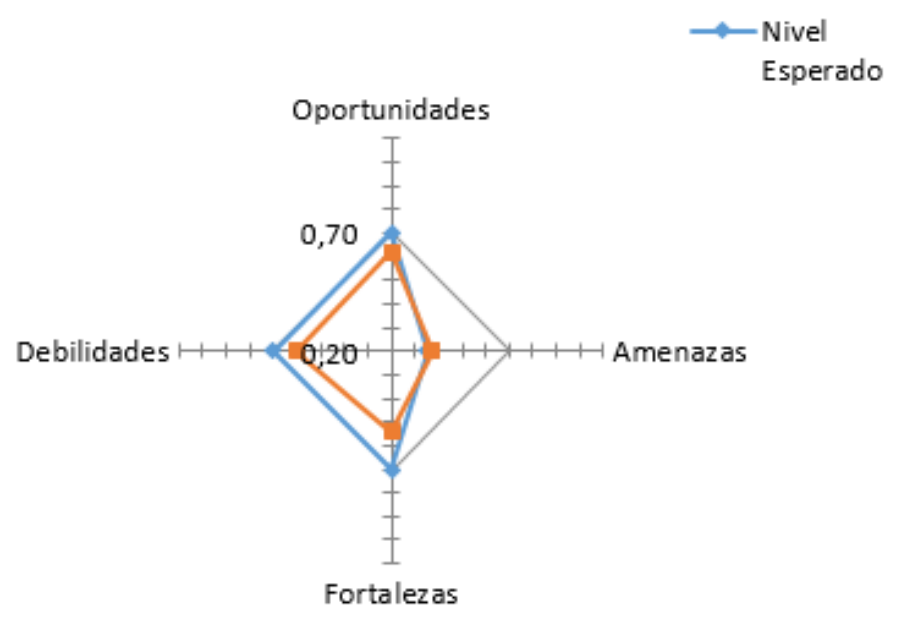

Figura 4. Análisis de brechas.

\section{Análisis de riesgos}

Luego del diagnóstico e identificación de riesgos es necesario aplicar una herramienta que permita visualizar el planteamiento del problema, los beneficios para los clientes internos y externos, los objetivos del proyecto y de esta manera poder mejorar la situación actual de la empresa a través de una planilla "six sigma" 
Revista Arbitrada Interdisciplinaria KOINONIA

Año 2020. Vol V. N³. Especial: Administración

Hecho el depósito de Ley: FA2016000010

ISSN: 2542-3088

FUNDACIÓN KOINONIA (F.K). Santa Ana de Coro. Venezuela.

Sebastián Mauricio Ormaza-Rodríguez; Johanna Rosalí Reyes-Reinoso; Fidel Edmundo Cepeda-Luna; Mireya Magdalena Torres-Palacios

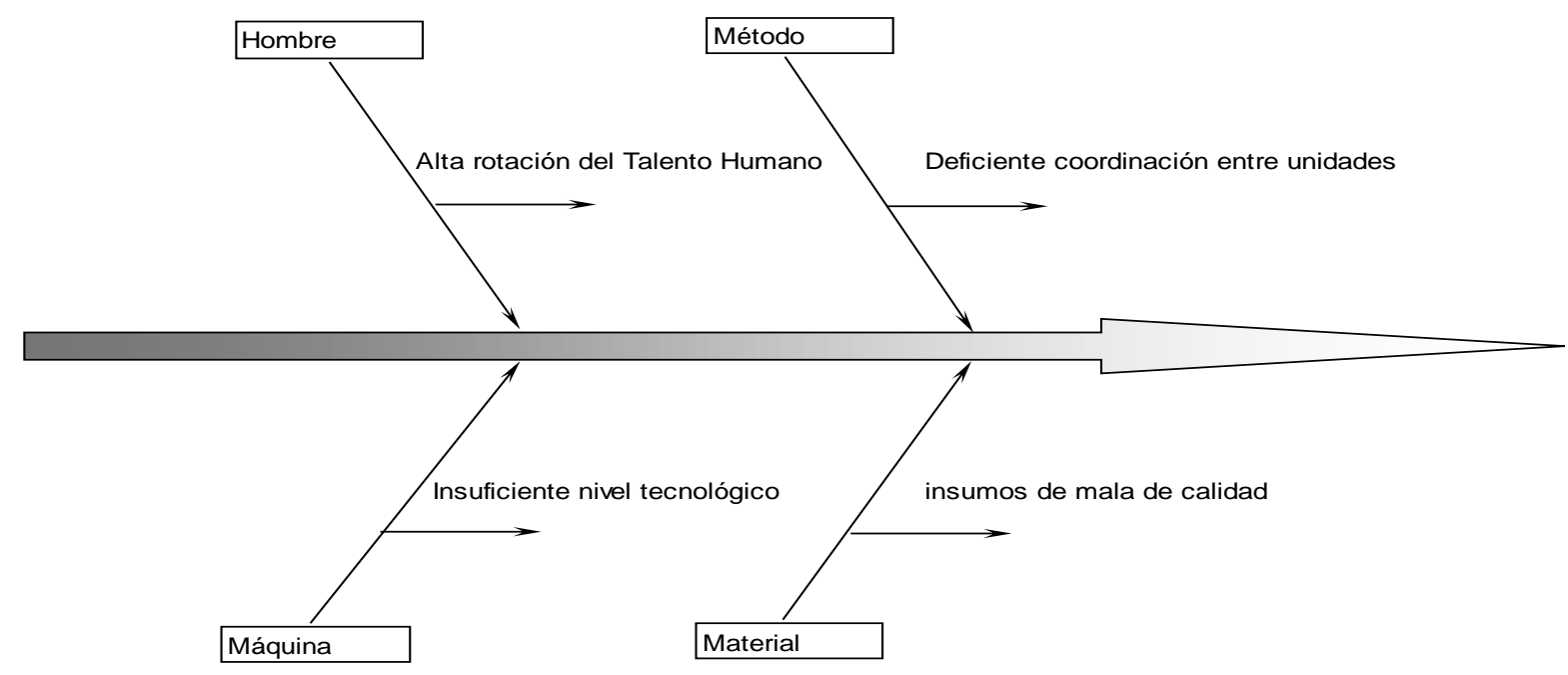

Figura 5. Fishbone aplicado a los riesgos identificados.

\section{$2^{2}$ Diseño factorial}

\begin{tabular}{|c|c|c|c|c|c|}
\hline \multirow[b]{2}{*}{$\mathbf{H}$} & \multirow[b]{2}{*}{ M } & \multicolumn{4}{|c|}{ Replications } \\
\hline & & MAT & 1 & 2 & 3 \\
\hline-1 & -1 & 1 & 28 & 25 & 27 \\
\hline 1 & -1 & -1 & 36 & 32 & 32 \\
\hline-1 & 1 & -1 & 18 & 19 & 23 \\
\hline 1 & 1 & 1 & 31 & 30 & 29 \\
\hline
\end{tabular}

$\begin{array}{lrrr}\text { ave }- & 23,3 & 30,0 & 26,7 \\ \text { ave }+ & 31,7 & 25,0 & 28,3 \\ \text { effect } & 8,3 & -5,0 & 1,7\end{array}$
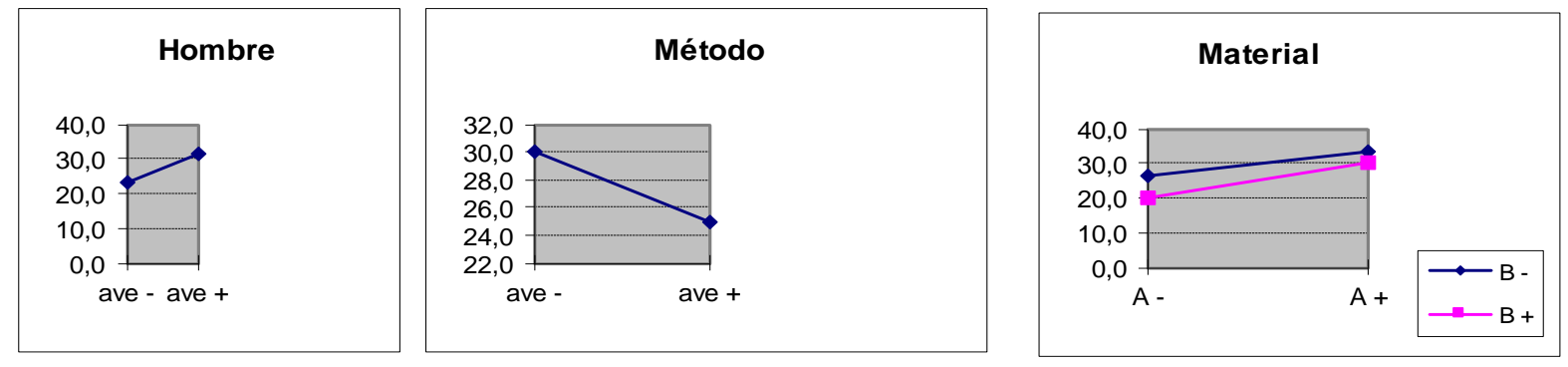

Figura 6. Diseño factorial a los riesgos identificados. 


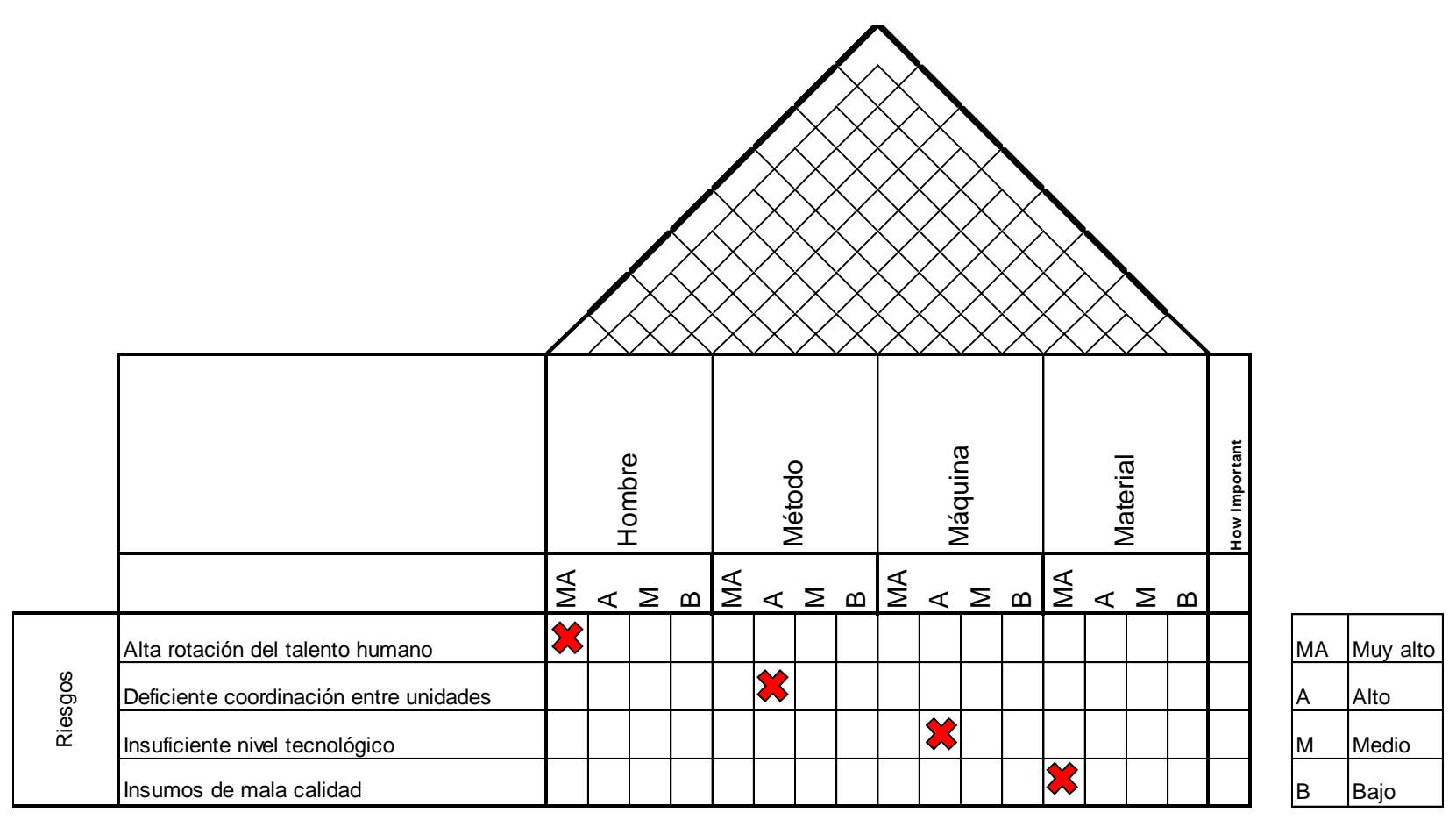

Figura 7. HOQ Six Sigma.

\section{Evaluación y control}

En este análisis que se realizó a la empresa EMAPAL EP, se pudo identificar las posibles amenazas y consecuencias que puede llevar a producir una demora en tiempo y de igual manera un retraso económico que afecta a la institución, ya que con los riesgos que se pudo reconocer, se puede evitar problemas con el cliente, así poder optimizar el tiempo, satisfacer a los usuarios de manera óptima, en la ejecución y control permitirá mejorar, ser eficientes y eficaces en los procesos para el bienestar de la cuidad de Azogues y sus zonas rurales y a su vez de manera interna en la empresa llevar un clima laboral con excelencia. 
Tabla 1.

Ejecución y control.

\section{EJECUCIÓN Y CONTROL}

\section{EJECUCIÓN}

Falta de coordinación entre departamentos para diferentes procesos

Alta rotación del talento humano que crea inconvenientes en toma de lecturas mensuales

Insuficiente nivel tecnológico que implica retraso en pago a los proveedores

Falta de sistematización de la empresa

\section{CONTROL}

-Controlar en tiempo real las actividades que se están realizando.

-implementar un sistema para coordinar procesos entre las diferentes áreas.

-Detectar y eliminar cuellos de botella.

-Disminuir los tiempos muertos entre actividades. -Reducir movimientos de documentos físicos e informáticos.

-Mejorar el sistema de software de los equipos de tomas de lectura.

-Capacitaciones continuas para el personal.

-Movilización para el personal para agilitar tiempo.

-Disminuir procesos repetitivos para agilitar tiempo.

- Contar con personal altamente capacitado para manejar el sistema financiero.

- Negociar con los proveedores para la obtención de descuentos por pagos anticipados.

-Establecer alertas o recordatorios de pago mediante sistemas avanzados de software.

- Contratar una mejor tecnología para el sistema de internet.

-Crear sistemas y/o programas para los distintos departamentos para facilitar los diferentes procesos.

-Mejorar el sistema de seguridad en las diferentes plantas de tratamiento de agua. 
Movilización restringida por falta de vehículos de propiedad de la empresa
-Obtención por comodato con otra empresa algunos vehículos.

-Disponer horarios para la utilizar los vehículos por los distintos departamentos.

\section{CONCLUSIONES}

Se establece una realidad y la importancia para la utilización de la evaluación de riesgos y gestión administrativa, por cuanto es una herramienta utilizada por las empresas y ayuda a detectar las debilidades, con ello se puede mejorar la calidad, un mejor desempeño para satisfacción de clientes internos y externos.

Se recomienda seguir los pasos analizados para obtener un aumento de rentabilidad, además para presentar un mejor clima laboral, agilitar procesos, mejoramiento en toma de lecturas, rapidez en pagos, desempeño mejorado en horarios de movilización y una mejor sistematización que ayuda notablemente a la organización.

La Empresa Pública Municipal de Agua Potable y Alcantarillado de Azogues puede controlar sus distintas actividades de una manera más eficiente y eficaz para así lograr quitar el "cuello de botella" que impedía a la empresa agilitar algunos procesos que eran muy necesarios para la satisfacción de sus clientes, proveedores y funcionarios.

\section{FINANCIAMIENTO}

No monetario.

\section{AGRADECIMIENTO}

A la directiva y funcionarios de la Empresa Municipal de Agua Potable, Alcantarillado y Saneamiento de Azogues (EMAPAL EP); por permitir y apoyar el desarrollo de la investigación. 


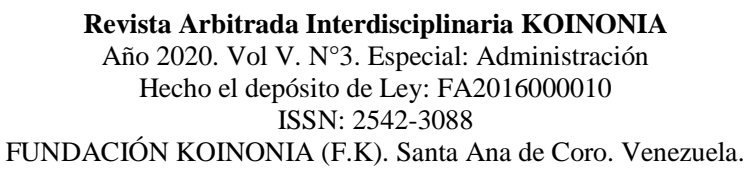

Sebastián Mauricio Ormaza-Rodríguez; Johanna Rosalí Reyes-Reinoso; Fidel Edmundo Cepeda-Luna; Mireya Magdalena Torres-Palacios

\section{REFERENCIAS CONSULTADAS}

Argudo-Tello, K. J., Erazo-Álvarez, J. C., \& Narváez-Zurita, C. I. (2019). Evaluación de Control Interno en Riesgos Estratégicos para la Dirección de Planificación de la Universidad de Cuenca. [Evaluation of Internal Control in Strategic Risks for the Planning Direction of the University of Cuenca]. Revista Arbitrada Interdisciplinaria Koinonía, 1(4), 67-96. http://dx.doi.org/10.35381/r.k.v4i1.372

Botero-Pinzón, L. (2017). El liderazgo en la gestión administrativa como impulsor de la estrategia para la competitividad internacional empresaria. Revista Ciencias Estratégicas, 25(38),271-276.

Calle, A. I., Narváez, C. I., \& Erazo, J. C. (2019). Auditoria en prevención de riesgos laborales y salud ocupacional: Procedimiento sistémico aplicado a la empresa Jasetrón. [ Audit in prevention of occupational risks and occupational health: Systemic procedure applied to the company Jasetrón]. Revista Arbitrada Interdisciplinaria Koinonía, 2(4), 25-55. http://dx.doi.org/10.35381/r.k.v4i2.466

Casas-Cervantes, A. (2015). La gestión comunitaria del agua y su relación con las políticas públicas municipales: El caso del manantial de Patamburapio en el estado de Michoacán, 2009-2014. [Community water management and its relationship with municipal public policies: The case of the Patamburapio spring in the state of Michoacán, 2009-2014]. Intersticios sociales, (10), 1-43.

Castaño-Ramírez, A. (2011). Planeación estratégica en la Pyme de Bogotá. Orientación al futuro y evasión de la incertidumbre. [Strategic planning in small and medium enterprises in Bogotá, Colombia Future-oriented approach and uncertainty prevention]. Contaduría y administración, (235), 147-173.

Diaz, E., \& León, M. (2019). Gestión Administrativa y comercial. [Administrative and commercial management]. Madrid: Paraninfo.

Empresa Pública del Agua EPA EP (2016). Plan estratégico empresarial 2017 - 2021 [Strategic business plan 2017 - 2021]. Recuperado de https://n9.cl/w58k2

Erazo-Álvarez, J. C., \& Narváez-Zurita, C. I. (2020). Medición y gestión del capital intelectual en la industria del cuero - calzado en Ecuador. [Measurement and management of intellectual capital in the leather industry - footwear in Ecuador]. Revista Arbitrada Interdisciplinaria Koinonía, 9(5)437-467. http://dx.doi.org/10.35381/r.k.v5i9.662 


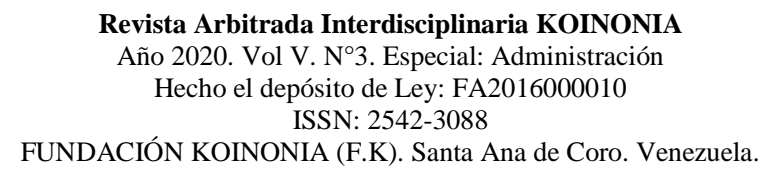

Sebastián Mauricio Ormaza-Rodríguez; Johanna Rosalí Reyes-Reinoso; Fidel Edmundo Cepeda-Luna; Mireya Magdalena Torres-Palacios

Galindo-Arévalo, L. V. (2019). Aproximación a los lineamientos para la implementación de la gestión del conocimiento en las entidades del sector público en Colombia. [Approach to the guidelines for the implementation of knowledge management in public sector entities in Colombia]. SIGNOS - Investigación En Sistemas De gestión, 12(1), 181-197. https://doi.org/10.15332/24631140.5428

Llanes-Guerra, O., Martínez-Massip, A., García-Pedraza, L., Zenea-Montejo, M., \& López-Verdecia, Y. (2019). Evaluación de responsabilidad social en cooperativas agropecuarias. [Evaluation of Social Responsibility in Agricultural Cooperatives]. Revista Estudios del Desarrollo Social: Cuba y América Latina, 7(3).

Medina-León, A., Nogueira-Rivera, D., Hernández-Nariño, A., \& Comas-Rodríguez, R. (2019). Procedimiento para la gestión por procesos: métodos y herramientas de apoyo. Ingeniare. Revista chilena de ingeniería, 27(2), 328342. https://dx.doi.org/10.4067/S0718-33052019000200328

Morón-Vásquez, A., \& Reyes-Matheus, M., \& Urbina-Chirinos, Á. (2015). Gestión de riesgos en la empresa R.C. Agelvis, C.A. [Risk management in the company R.C. Agelvis, C.A.]. Multiciencias, 15(4),417-427.

Murillo-Vilela, C. H., Erazo-Álvarez, J. C., Quevedo-Vázquez, J. O., \& Narváez Zurita, C. I. (2019). Plan de inversión como herramienta de la gestión del capital intelectual. [Investment plan as a tool for intellectual capital management]. Revista Arbitrada Interdisciplinaria Koinonía, 1(4), 245-273. http://dx.doi.org/10.35381/r.k.v4i1.458

Orellana-Palomeque, D. P., Erazo-Álvarez, J. C., Narváez-Zurita, C. I., \& MatovelleRomo, M. M. (2019). Gestión administrativa, elemento clave para el desarrollo de las empresas de servicio de transporte. [Administrative management, key element for the development of transport service companies]. Visionario Digital, 3(2.2), 238-260.

Reyes, P. E., Narváez, C. I., Erazo, J. C., \& Giler, L. V. (2020). Configuración del impuesto a la patente municipal con base al ingreso de las actividades económicas. Caso: GAD Municipal de Pucará - Ecuador. [Configuration of the municipal license tax based on income from economic activities. Case: Municipal GAD of Pucará Ecuador]. Revista Espacios, 41(21), 197-211.

Sanz, S., \& López, J. (2018). Gestión Administrativa. [Administrative management]. Madrid: Impresos y revistas. 
Sebastián Mauricio Ormaza-Rodríguez; Johanna Rosalí Reyes-Reinoso; Fidel Edmundo Cepeda-Luna; Mireya Magdalena Torres-Palacios

Torres-Navarro, C., Malta-Callegari, N., Zapata-Casabón, C., \& Aburto-Viveros, V. (2015). Metodología de gestión de riesgo para procesos en una institución de salud previsional. [Risk management methodology for processes in a pension health institution]. Universidad, Ciencia y Tecnología, 19(75), 98-109.

Trógolo, M, Pereyra, A., \& Sponton, C.. (2013). Impacto de Diferentes Estilos de Liderazgo Sobre el Engagement y Burnout: Evidencia en una Muestra de Trabajadores Argentinos. [Impact of different leadership styles on work engagement and burnout: evidence from a sample of argentine workers]. Ciencia \& trabajo, 15(48), 152-157. https://dx.doi.org/10.4067/S0718$\underline{24492013000300008}$

Varela-Valenzuela, L. (2013). Prevención de riesgos. [Risks prevention]. Ciencia \& trabajo, 15(47), a1. https://dx.doi.org/10.4067/S0718-24492013000200001 